\title{
Original
}

\section{Characterization of SSEA-3 Positive Cells Derived from Human Dental Pulp Stem Cells}

\author{
Takatoshi Nagano, Taichiro Funatsu and Kazuhiro Gomi
}

Department of Periodontology, Tsurumi University School of Dental Medicine, Kanagawa, Japan

(Accepted for publication, August 23, 2019)

\begin{abstract}
Mesenchymal stem cells are known to exist in pulp tissue. However, the detailed characteristics of the stem cells have not been elucidated. Recently, the presence of novel Muse cells which has been reported. Researches mentioned that Muse cells exist in all mesenchymal tissues such as in the bone marrow, skin and adipose tissue. However, there is hardly any report regarding the existence of Muse cells in oral tissues. Therefore, the purpose of the study was to determine the presence of Muse cells in dental pulp tissue. About $0.5 \%$ of SSEA (Stage-Specific Embryonic Antigen)-3 positive cells were isolated from dental pulp cells by cell sorter. The isolated cells showed self-renewal ability in addition to expressing the genes representing the three germ layers. Furthermore, the isolated cells showed a high calcification ability when subjected to calcification-inducing medium. Calcification ability was shown using alkaline phosphatase staining, alizarin red staining and quantification of calcium. The results suggest that SSEA-3 positive cells derived from human dental pulp tissue are similar to Muse cells, highly capable of differentiating into osteogenic cells. Isolated these cells from the dental pulp tissue is highly advantageous since they are less invasive compared to other cells. Hence, the dental pulp tissue is envisioned to be the source of new cells beneficial in regenerative medicine.
\end{abstract}

Key words: Dental pulp, SSEA-3, Flow cytometry

\section{Introduction}

Currently, researches on stem cells for regenerative medicine are highly advancing. Studies on ES cells ${ }^{1,2)}$, iPS cells ${ }^{3,4)}$ and other cells used in regenerative medicine are making progress. In 2011, mesenchymal cells positive to both SSEA-3 and CD105, manifested their ability to differentiate into various cells of the body just like iPS cells and ES cells. These cells were called Muse cells ${ }^{5)}$. One characteristic of Muse cells is their non-tumorigenicity unlike iPS cells or other stem cells ${ }^{5,6)}$. Muse cells are transplanted via blood vessels and accumulate in injured sites. Studies mentioned that Muse cells spontaneously differentiate into cells in response to tissue repair and regeneration of damaged tissues ${ }^{5,7)}$, thus, Muse cells can be utilized in advanced regenerative medicine. Since Muse cells are mesenchymal in origin, a percentage of those cells can be found on any tissue in the body. Thus far, major studies revealed that Muse cells can be harvested from bone marrow, skin, adipose tissues $^{5,8-12)}$. Notwithstanding, only a few studies showed the presence of Muse cells in oral tissues collected from the periodontal ligament ${ }^{13)}$.

Gronthos et al. ${ }^{14)}$ published a study on the existence of stem cells in pulp tissue. They found out that the odontoblasts that differentiated from dental pulp stem cells possess various characteristics such as self-renewal and differentiation ${ }^{15}$. Subsequent studies mentioned the differentiation of dental pulp stem cells into nerve cells, adipocytes, chondrocytes, etc, as well as during bone formation ${ }^{16-22)}$. Moreover, the use of dental pulp stem cells is not limited to Dentistry in terms of dentin and bone regeneration. In fact, its application in systemic diseases has been reported. Arthur et al suggested that it is possible for dental pulp stem

Correspondence to: Dr. Takatoshi Nagano, Department of Periodontology, Tsurumi University School of Dental Medicine, 2-1-3 Tsurumi, Tsurumi-ku, Yokohama, 230-8501, Japan; Tel: +81-45-580-8434; Fax: +81-45-573-9599; e-mail: nagano-takatoshi@tsurumi-u.ac.jp cells to differentiate into cells having neural functions, thus can be applied to treat neurological disorders ${ }^{23}$. Furthermore, Ikeda et al suggested that dental pulp stem cells have a superior proliferative capability and that damaged liver tissues can recuperate with its application ${ }^{24)}$. As described above, dental pulp cells are very promising cells for regenerative medicine yet no report on Muse cells isolated from dental pulp tissue was found. Therefore, in this study, we aimed to isolate and characterize cells from human dental pulp stem cells (HDPSC) having similar capabilities with Muse cells.

\section{Cells}

\section{Materials and Methods}

Commercial dental pulp cells (HDPSC, Lonza, Inc., Basel, Switzerland) were used in the experiment with the approval of the ethics committee (Ethics Committee approval number: 1113).

\section{Cell culture}

Dulbecco's Modified Eagle Medium (DMEM, Life Technologies, Inc., Carlsbad, CA, USA) was the growth medium used supplemented with $10 \%$ fetal bovine serum (FBS, Sigma-Aldrich, Co., St. Louis, MO, USA) and $0.1 \mathrm{mg} / \mathrm{ml}$ kanamycin (Life Technologies, Inc., CA, USA). HDPSC, $3.0 \times 10^{5}$, were seeded in T75 flasks and cultured in an incubator under $5 \% \mathrm{CO}_{2}$ condition. The medium was changed every 72 hours. Upon reaching subconfluence, exfoliation and passage of the cells were performed using Trypsin-EDTA (Life Technologies, Inc., Carlsbad, CA, USA). Cells were cultured until reaching over confluence and used for sorting after three passages.

\section{Flow cytometry and cell sorting}

Upon reaching over confluence at the 3rd passage, HDPSC were de- 
tached and harvested using Trypsin-EDTA. Anti-SSEA-3 (Biolegend, Inc., San Diego, CA, USA) was used as the primary antibody. Staining was performed using FITC conjugated anti-rat IgM (Jackson ImmunoResearch, Inc., West Grove, PA, USA) as the secondary antibody. EDTA-2Na of 2mM (Nacalai Tesque, Inc., Japan) was added to phosphate buffered saline (PBS, Sigma-Aldrich, Co., St. Louis, MO, USA). Sorting was performed with a flow cytometer (Sony, Co., Tokyo, Japan) using FACS buffer prepared by adding $0.5 \%$ bovine serum albumin (BSA, Nacalai Tesque, Inc., Japan).

\section{Observation of cell growth}

In order to observe the proliferation ability of SSEA-3 positive cells obtained by sorting, $1.0 \times 10^{4}$ cells were seeded on a 6 -well plate and cultured using a growth medium. The medium was changed every 72 hours. Cell counting was carried out for 10 days immediately after sowing with a 24-hour interval.

\section{Observation of cluster formation and self-renewal ability}

Cells sorted from HDPSC were cultured in poly 2-hydroxymethyl methacrylate (Sigma-Aldrich, Co., St. Louis, MO, USA) on a 96-well plate. The medium was added once every 3 days and floating culture was performed. Clusters were identified as a group of cells having a diameter of $25 \mu \mathrm{m}$ or more. Cluster formation from individual cells was observed for 5 to 7 days after seeding. Formed clusters were then collected and seeded on a gelatin-coated 24-well plate for cell adhesion culture. RNA was harvested from the attached, proliferated cells and those individual cells were resuspended to determine their capability of forming clusters.

The ability of the cells to self-replicate was assessed by observing whether individual cells maintained in resuspension culture would form clusters after attaching and culturing from clustered cells.

\section{Gene expression representing the three germ layers (Quantitative PCR: Q-PCR) \\ Total RNA was collected using Nucleo spin RNA XS (Mache- rey-Nagel, GmbH \& Co., KG., Duren, Germany), and reverse transcrip- tion to cDNA was performed using SuperScript VILO cDNA Synthesis Kit (Life Technologies, Inc., Carlsbad, CA, USA). PCR was carried out using FastStart Essential DNA Green Master (Roche, Ltd., Basel, Swit- zerland). The expression of hGAPDH (an endogenous control), $\alpha$-feto- protein (AFP) and GATA6 (endodermal markers), Brachyury (a meso- dermal marker) and Nestin (an ectodermal marker) were tested. Each primer design is shown on Table 1.}

\section{Calcification induction (Alkaline Phosphatase [ALP] staining, Aliza- rin red staining, Ca quantification)}

Calcification was induced on cells sorted from adhered cells. Cell count of $3.0 \times 10^{4}$ was seeded on a 6 -well plate, cultured in a growth medium and placed in an incubator at $37^{\circ} \mathrm{C}$ and under $5 \% \mathrm{CO}_{2}$. After adhesion was confirmed the next day, calcification medium (10\% FBS, $10 \mathrm{nmol} / 1$ dexamethasone, $0.05 \mu \mathrm{mol} / 1$ ascorbic acid, $10 \mu \mathrm{mol} / 1$ beta sodium glycerol phosphate added DMEM) was added and replaced every 72 hours. ALP and alizarin red stainings were performed at week 1, 2 and 3 after inducing calcification and the amount of $\mathrm{Ca}$ deposited was determined.

\section{ALP staining}

Cells on a 6-well plate, subjected to calcification after 1, 2 and 3 weeks were stained with ALP. After removing the culture supernatant,

\begin{tabular}{ccc}
\multicolumn{2}{c}{ Table 1. PCR primers } \\
\hline Gene & & Primer Sequence 5' $\rightarrow 3$ \\
\hline \multirow{2}{*}{ hGAPDH } & Sence & :ATCAAGAAGGTGGTGAAGCA \\
& Antisence & :GTCGCTGTTGAAGTCAGAGGA \\
\hline \multirow{2}{*}{$\alpha$-fetoprotein } & Sence & :CCACTTGTTGCCAACTCAGTGA \\
& Antisence & :TGCAGGAGGGACATATGTTTCA \\
\hline \multirow{2}{*}{ GATA6 } & Sence & :CCTGCGGGCTCTACAGCAAGATGAAC \\
& Antisence & AAGGGTGCAGGAGACACAGATAC \\
\hline \multirow{2}{*}{ Brachyury } & Sence & :GAACAGCTCTCCAACCTATG \\
& Antisence & :AGACTGGGATACTGGCTAGAG \\
\hline \multirow{2}{*}{ nestin } & Sence & :TGCGGGCTACTGAAAAGTCC \\
& Antisence & :TGTAGGCCCTGTTTCTCCTG \\
\hline
\end{tabular}

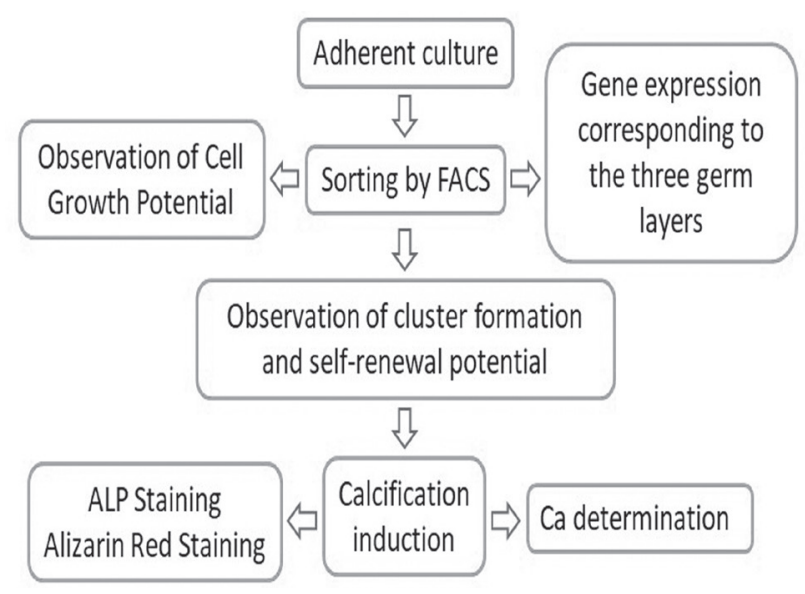

Figure 1. Experiment flowchart

the cells were washed with PBS, fixed with $100 \%$ methanol and then washed again with PBS three times. Subsequently, the adjusted substrate solution was added to each well and allowed to react for 30 minutes at $37^{\circ} \mathrm{C}$. Thereafter, the cells were washed again with PBS three times, dried and finally, the staining was assessed.

\section{Alizarin red staining}

Cells on a 6-well plate subjected to calcification after 1, 2 and 3 weeks were subjected to Alizarin red S staining. After removing the culture supernatant, the cells were washed with PBS, fixed with $100 \%$ ethanol, and then washed again with distilled water three times. Next, $1.0 \%$ alizarin red S staining solution was added and the mixture was allowed to react for 10 minutes at room temperature. Thereafter, the samples were washed again with distilled water three times, dried and the staining was assessed.

\section{Ca quantification}

The amount of calcium formed on a 6-well plate was measured at 1 , 2 and 3 weeks after induction. After removing the culture supernatant, the cells were washed with PBS and adherent cells were lysed using 0.5 $\mathrm{M}$ hydrochloric acid. The suspension was used for measurement. Calcium E-Test (Wako Pure Chemical Industries, Ltd., Osaka, Japan) was performed where samples were placed on a plate reader at a wavelength of $570 \mathrm{~nm}$ to calculate the amount of calcium.

Fig. 1 shows the outline of the experiment. 


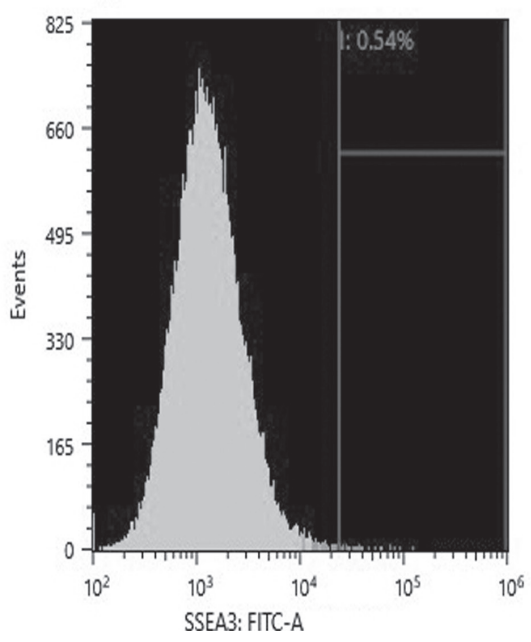

G

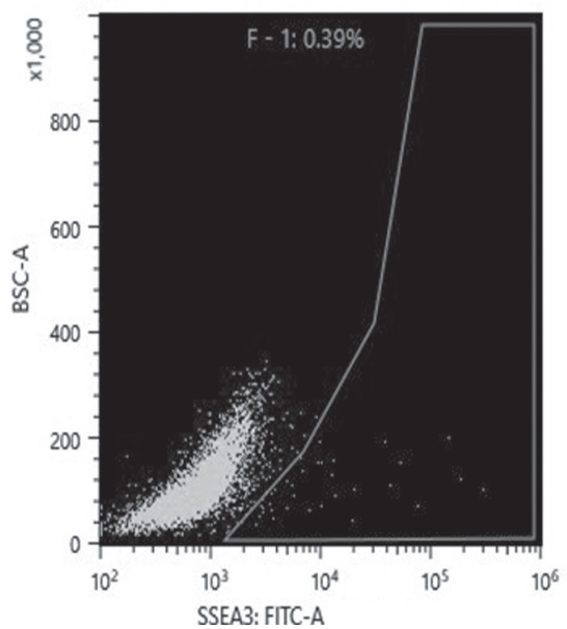

Figure 2. Preparation of SSEA-3 positive cells from HDPSC. About 0.5\% SSEA-3 positive cells were isolated from HDPSC.

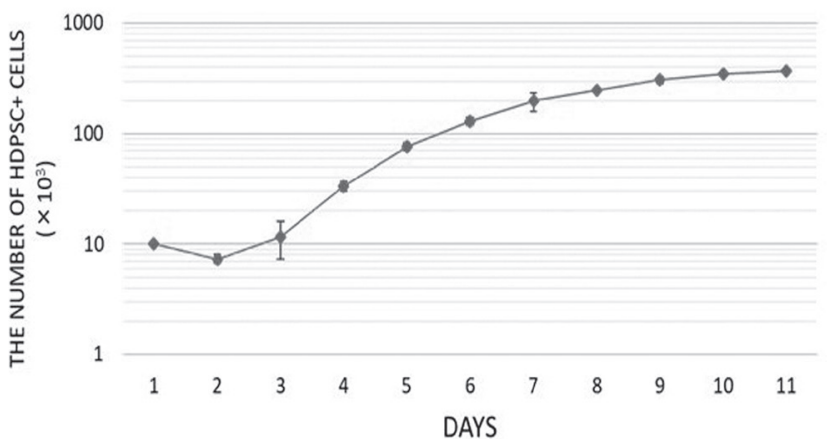

Figure 3. Cell proliferation ability of HPDSC-derived SSEA-3 positive cells. When the HDPSC-derived SSEA-3 positive cells were cultured in a 6-well plate, proliferation ability similar to typical mesenchymal cells was evident and the confluence was reached 10 days after seeding.

\section{Results}

\section{Flow cytometry and cell sorting}

HDPSC that reached over confluency were stained with anti-SSEA-3 antibody and then sorted using FITC. About $0.5 \%$ of SSEA- 3 positive cells were isolated from HDPSC (Fig. 2).

\section{Observation of cell growth potential}

Fig. 3 shows the growth curve drawn for 10 days of sowing the HPDSC-derived SSEA-3 positive cells. The graph shows the increasing growth potential of the isolated cells.

\section{Observation of cluster formation and self-renewal ability}

The SSEA-3 positive cells sorted from HDPSC were floated and cultured on a poly-HEMA coated 96-well plate for each individual cell. Clusters exceeding $25 \mu \mathrm{m}$ in diameter were examined (Fig. 4A). Clusters were adhered on gelatin-coated dishes and then detached and seeded

\section{(A)}
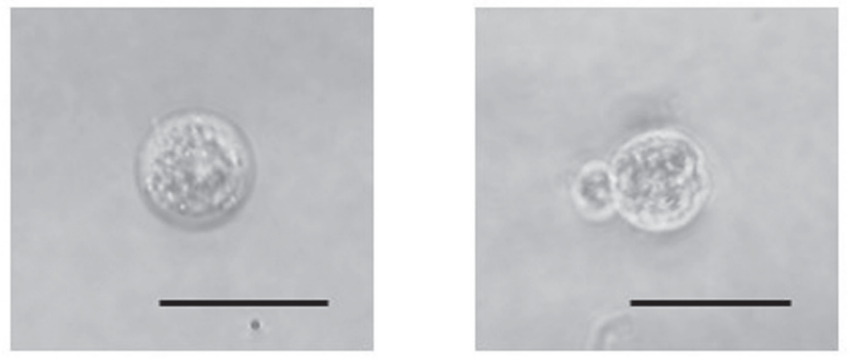

Scale bar: $50 \mu \mathrm{m}$

(B)
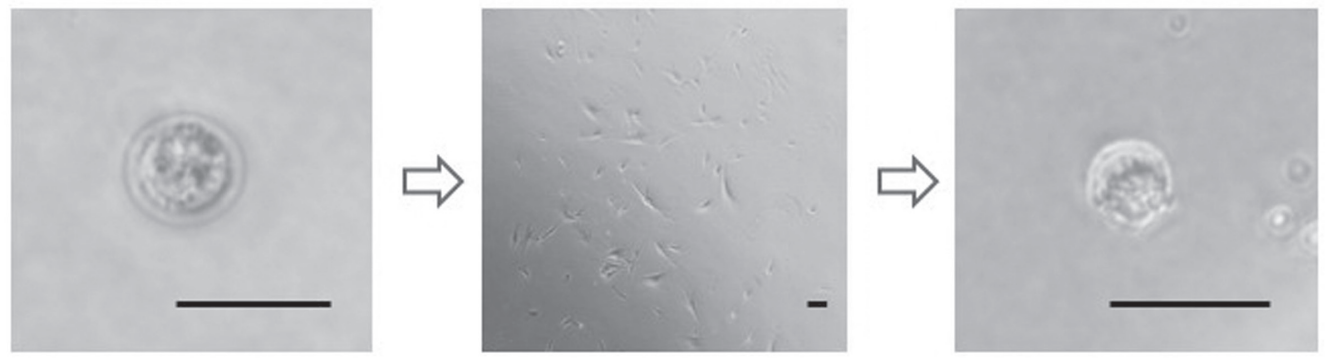

Figure 4. Cluster formation and observation of self-renewal ability of HDPSC-derived SSEA-3 positive cells. HDPSC-derived SSEA-3 positive cells isolated from cell sorting were floated and cultured on a poly-HEMA coated 96-well plate individually. Cells were able to form clusters greater than $25 \mu \mathrm{m}$ in diameter(A). These clusters adhered and proliferated on gelatin-coated dishes. The clusters were separated and allowed to grow again individually in poly-HEMA coated 96-well plate after seeding(B). Scale bar: $50 \mu \mathrm{m}$. 


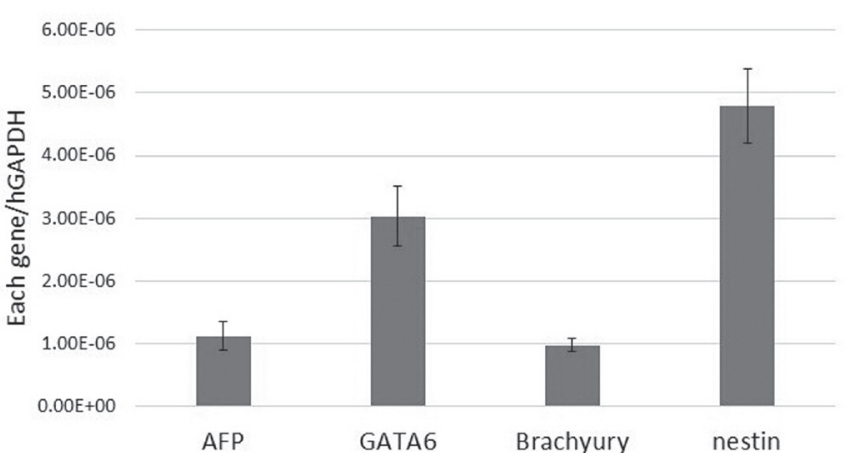

Figure 5. Observation of three embryonic gene expression of HDPSC-derived SSEA-3 positive cells. For verification of pluripotency, gene expression representing the three germ layers was analyzed by quantitative-PCR The isolated cells expressed the genes representing the three germ layers ( $\alpha$-fetoprotein [AFP], GATA6, Brachyury, nestin). Mean \pm standard error $(\mathrm{n}=3)$.

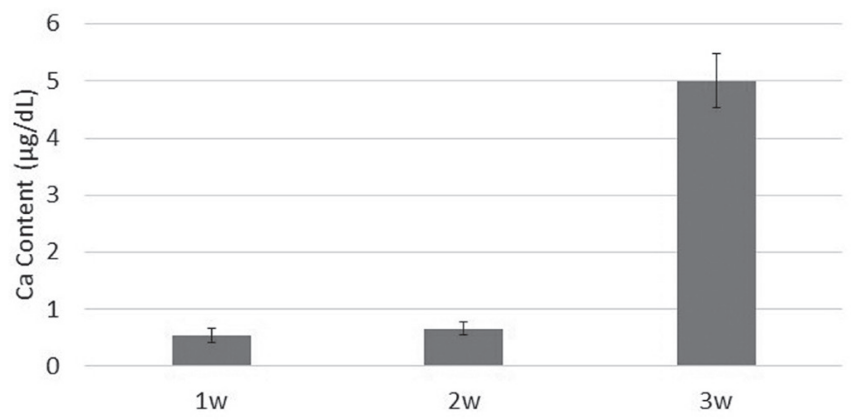

Figure 7. Calcium quantification of HDPSC-derived SSEA-3 positive cell at 1,2 and 3 weeks after calcification induction. Mean \pm standard error $(n=3)$. The amount of calcium was measured at 1,2 and 3 weeks after initiation of calcification in HDPSC+ cultured in a 6-well plate. Calcium concentration increased over time.

once more on a poly-HEMA coated 96-well plate to determine its capability to form clusters again. Cultured individual cells again were able to form clusters indicating their self-renewal ability (Fig. 4B).

\section{Gene expression representing the three germ layers (Q-PCR)}

Verification of pluripotency was carried out by analyzing the gene expression representing the three germ layers by Q-PCR based on RNA collected from SSEA-3 positive cells sorted from HDPSC. Results showed that all genes representing the three germ layers were expressed. Particularly, Nestin, which is a gene expressed by the ectodermal cells, was strongly expressed (Fig. 5).

\section{Calcification induction (ALP staining, Alizarin red staining, Ca quan- tification) \\ ALP staining}

SSEA-3 positive cells on a 6-well plate were cultured in a calcification medium followed by staining with ALP at 1, 2 and 3 weeks after induction. Intense ALP staining was confirmed on HDPSC-derived SSEA3 positive cells (Fig. 6A).

\section{Alizarin red staining}

SSEA-3 positive cells on a 6-well plate were cultured in a calcification medium followed by staining with alizarin red at 1,2 and 3 weeks after induction. Intense alizarin red was observed on HDPSC-derived
(A)

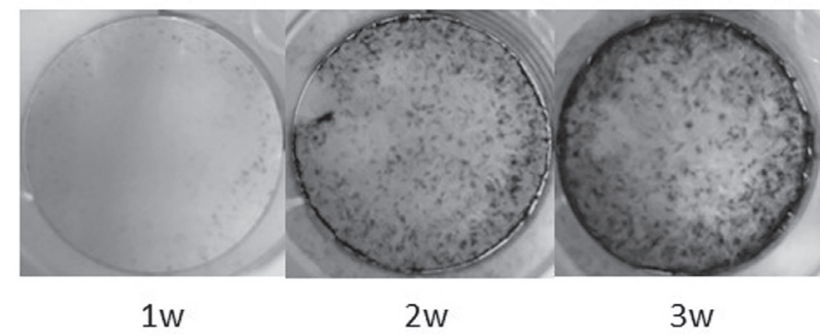

(B)

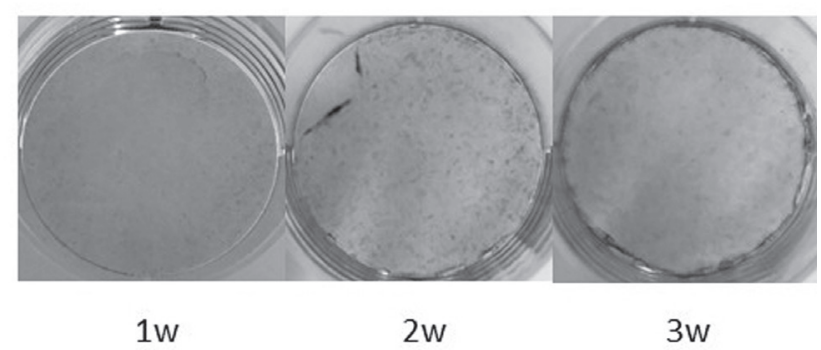

Figure 6. ALP and alizarin red stained images at 1,2 and 3 weeks after mineralization induction in HDPSC-derived SSEA-3 positive cells. (A) ALP staining (B) Alizarin red. Staining for both ALP and alizarin red increased over time.

SSEA-3 positive cells and the intensity progressed over time. Calcified substances were clearly stained at 3 weeks confirming its high calcification ability (Fig. 6B).

\section{Ca quantification}

We measured the amount of calcium at 1, 2 and 3 weeks after inducing calcification on SSEA-3 positive cells cultured on a 6-well plate (Fig. 7). Calcium concentration increased over time and prominent calcium production was confirmed 3 weeks after calcification induction.

\section{Discussion}

Mesenchymal stem cells are present in soft tissues in the oral cavity and it is thought that these stem cells have functional roles on periodontal tissue regeneration using materials such as GTR or enamel matrix protein. It is known that stem cells positive to STRO-1, SSEA-4 and MUC-18 are present in oral tissues but the existence of pluripotent Muse cells that are positive to SSEA-3 and CD105 is uncertain ${ }^{5,8-12)}$.

The ability of Muse cells to form clusters all over again from individual cells on floating culture has been recognized ${ }^{5}$. In this experiment, about $0.5 \%$ SSEA-3 positive cells were sorted out from HDPSC. The proliferation ability of the isolated cells was almost similar to the SSEA3 positive cells harvested from the periodontal ligament and skin ${ }^{13}$ showing the same growth curve exemplified by typical undifferentiated mesenchymal cells. In addition, cluster formation was observed when individual cells were cultured on a suspension medium. Self-renewal ability was confirmed when cells from the original clusters were separated and then were able to form clusters for the second time. Furthermore, Q-PCR was carried out to determine the gene expression representing the three embryonic layers. Endodermal markers such as AFP and GATA6, mesodermal marker Brachyury, and ectodermal marker Nestin were all expressed. Based on the results, the isolated cells are considered to be Muse cells present in HDPSC. We thought that many mesenchymal stem cells are contained in the dental pulp tissue and so 
we considered that a number of Muse cells would also be isolated from HDPSC. However, current result showed a lower percentage of Muse cells isolated from HDPSC than those isolated from human periodontal ligament fibroblasts (HPdLF) and human normal skin fibroblasts (HNHDF) reported earlier ${ }^{13)}$. This was thought to be due to the fact that the dental pulp cells consist of a more differentiated cell population unlike the Muse cells which are more undifferentiated.

Mineralization was induced in HDPSC-derived SSEA-3 positive cells to determine the ability to produce calcium. Strong ALP and alizarin red stainings were obtained at 1,2 and 3 weeks after induction and the intensity increased over time indicating that HDPSC-derived SSEA3 positive cells have high calcification ability. Similar results were obtained when the amount of calcium deposition was measured. Calcification is highly possible to take place since HDPSC is originally responsible for dentin repair and regeneration. Thus differentiation into cell capable of producing hard tissues is high probable.

The application of pluripotent Muse cells in early regenerative medicine as is highly expected. Currently, Muse cells transported in the bloodstream, tend to accumulate, proliferate and differentiate to participate in repair and regeneration of damaged tissues. Whether those cells are part of the damaged tissue or sorted from similar tissues, Muse cells can effectively aid in tissue regeneration. In this study, we characterized the SSEA-3 positive cells and based on our preliminary study, most SSEA-3 positive cells are also positive to $\mathrm{CD} 105^{13}$. By decreasing the sorting process, cell damage could have been avoided thus increasing the count of isolated undifferentiated mesenchymal cells. Teeth extracted for justifiable reasons contain organized tissues such as pulp and periodontal ligament and discarding them is considered medical waste. Currently, regenerative medicine using bone marrow cells is mainly performed. Nevertheless, new cells can be harvested from the pulp which are considered less invasive compared to bone marrow cells. Thus, dental pulp cells are effective source of new cells in the future.

If the cells to be used in regenerative medicine have sufficient phenotype of their source, then those cells can be efficiently isolated and regenerated to develop the corresponding body tissues. Establishing the right flow or process from cell sorting to transplantation is an important step for efficient regenerative medicine.

\section{Acknowledgements}

This study was supported by a JSPS KAKENHI Grant-in-Aid for Scientific Research (16K11844).

\section{Conflict of Interest}

The authors declare no conflict of interest with the companies and organizations, etc in relation to the study.

\section{References}

1. Evans MJ and Kaufman MH. Establishment in culture of pluripotential cells from mouse embryos. Nature 292: 154-156, 1981

2. Thomson JA, Itskovitz-Eldor J, Shapiro SS, Waknitz MA, Swiergiel JJ, Marshall VS and Jones JM. Embryonic stem cell lines derived from human blastocysts. Sience 282: 1145-1147, 1998

3. Takahashi K and Yamanaka S. Induction of pluripotent stem cells from mouse embryonic and adult fibroblast cultures by defined factors. Cell 126: 663-676, 2006

4. Takahashi K, Tanabe K, Ohnuki M, Narita M, Ichisaka T, Tomoda $\mathrm{K}$ and Yamanaka S. Induction of pluripotent stem cells from adult human fibroblasts by defined factors. Cell 131: 861-872, 2007

5. Kuroda Y, Kitada M, Wakao S, Nishikawa K, Tanimura Y, Mak- inoshima H, Goda M, Akashi H, Inutsuka A, Niwa A, Shigemoto T, Nabeshima Y, Nakahata T, Nabeshima Y, Fujiyoshi Y and Dezawa M. Unique multipotent cells in adult human mesenchymal cell populations. Proc Natl Acad Sci USA 107: 8639-8643, 2010

6. Wakao S, Kitada M and Dezawa M. The elite and stochastic model for iPS cell generation: multilineage-differentiating stress enduring (Muse) cells are readily reprogrammable into iPS cells. Cytometry A 83: 18-26, 2013

7. Wakao S, Kuroda Y, Ogura F, Shigemoto T and Dezawa M. Regenerative effects of mesenchymal stem cells: contribution of muse cells, a novel pluripotent stem cell type that resides in mesenchymal cells. Cells 1: 1045-1060, 2012

8. Wakao S, Kitada M, Kuroda Y, Shigemoto T, Matsuse D, Akashi H, Tanimura Y, Tsuchiyama K, Kikuchi T, Goda M, Nakahata T, Fujiyoshi Y and Dezawa M. Multilineage-differentiating stress-enduring (Muse) cells are a primary source of induced pluripotent stem cells in human fibroblasts. Proc Natl Acad Sci USA 108: 9875-9880, 2011

9. Kuroda Y, Wakao S, Kitada M, Murakami T, Nojima M and Dezawa M. Isolation, culture and evaluation of multilineage-differentiating stress-enduring (Muse) cells. Nat Protoc 8: 1391-1415, 2013

10. Heneidi S, Simerman AA, Keller E, Singh P, Li X, Dumesic DA and Chazenbalk G. Awakened by cellular stress: isolation and characterization of a novel population of pluripotent stem cells derived from human adipose tissue. PLoS One 8: e64752, 2013

11. Tsuchiyama K, Wakao S, Kuroda Y, Ogura F, Nojima M, Sawaya N, Yamasaki K, Aiba S and Dezawa M. Functional melanocytes are readily reprogrammable from multilineage-differentiating stress-enduring (muse) cells, distinct stem cells in human fibroblasts. J Invest Dermatol 133: 2425-2435, 2013

12. Ogura F, Wakao S, Kuroda Y, Tsuchiyama K, Bagheri M, Heneidi S, Chazenbalk G, Aiba S and Dezawa M. Human adipose tissue possesses a unique population of pluripotent stem cells with nontumorigenic and low telomerase activities: Potential implications in regenerative medicine. Stem Cells Dev 23: 717-728, 2014

13. Funatsu T, Gomi K, Matsushima Y, Ujiie Y and Nagano T. Characterization of mesenchymal stem cells derived from periodontal ligament. J Hard Tissue Biol 27: 131-138, 2018

14. Gronthos S, Mankani M, Brahim J, Robey PG, and Shi S. Postnatal human dental pulp stem cells (DPSCs) in vitro and in vivo. PNAS 97: 13625-13630, 2000

15. Gronthos S, Brahim J, Li W, Fisher LW, Cherman N, Boyde A, DenBesten P, Robey PG, and Shi S. Stem cell properties of human dental pulp stem cells. J Dent Res 81: 531-535, 2002

16. Iohara K, Zheng L, Ito M, Tomokiyo A, Matsushita K, and Nakashima M. Side population cells isolated from porcine dental pulp tissue with self-renewal and multipotency for dentinogenesis, chondrogenesis, adipogenesis, and neurogenesis. Stem Cells 24: 2493-2503, 2006

17. Jo YY, Lee HJ, Kook SY, Choung HW, Park JY, Chung JH, Choung YH, Kim ES, Yang HC, and Choung PH. Isolation and characterization of postnatal stem cells from human dental tissues. Tissue Eng 13: 767-773, 2007

18. Graziano A, d'Aquino R, Laino G and Papaccio G. Dental pulp stem cells: a promising tool for bone regeneration. Stem Cell Rev 4: 21 26, 2008

19. Yamada Y, Nakamura S, Ito K, Sugito T, Yoshimi R, Nagasaka T and Ueda M. A feasibility of useful cell-based therapy by bone regeneration with deciduous tooth stem cells, dental pulp stem cells, 
or bone-marrow-derived mesenchymal stem cells for clinical study using tissue engineering technology. Tissue Eng Part A 16: 18911900, 2010

20. Fujii Y, Kawase-Koga Y, Hojo H, Yano F, Sato M, Chung U, Ohba $\mathrm{S}$ and Chikazu D. Bone regeneration by dental pulp stem cells using a helioxanthin derivative and cell-sheet technology. Stem Cell Research \& Terapy 9: 24, 2018

21. Fukushima T, Tatehara S, Takabe Y, Tokuyama-Toda R and Satomura K. Dental pulp stem cell-derived, scaffold-free cinstructs for bone regeneration. Int J Mol Sci 19: 1846, 2018

22. Cinelli J, Nguyen E and Kingsley K. Assessment of dental pulp stem cell (DPSC) biomarkers following induction with bone morphogenic protein 2 (BMP-2). JABB 19: 1-12, 2018

23. Arthur A, Rychkov G, Shi S, Koblar SA and Gronthos S. Adult human dental pulp stem cells differentiate toward functionally active neurons under appropriate environmental cues. Stem Cells 26: 1787-1795, 2008

24. Ikeda E, Yagi K, Kojima M, Yagyuu T, Ohshima A, Sobajima S, Tadokoro M, Katsube Y, Isoda K, Kondoh M, Kawase M, Go MJ, Adachi H, Yokota Y, Kirita T and Ohgushi H. Multipotent cells from the human third molar: feasibility of cell-based therapy for liver disease. Differentiation 76: 495-505, 2007 